\title{
Influencia del estilo de vida, el sexo, la edad y el imc sobre la salud física y psicológica en jóvenes universitarios
}

\author{
Influence of Lifestyle, Gender, fige and Bmı on Physical and Psychological \\ Health in University Young \\ Influência do estilo de vida, o sexo, a idade e 0 Imc sobre a saúde física \\ e psicológica em jovens universitários
}

\author{
Luisa Teresa Angelucci*, Yolanda Cañoto, María Jimena Hernández \\ Universidad Católica Andrés Bello en Caracas
}

Doi: http://dx.doi.org/10.12804/revistas.urosario.edu.co/apl/a.4454

\section{Resumen}

Los posibles cambios en los estilos de vida que se producen al ingresar en la universidad, en conjunto con otros factores, impactan la salud, tanto física como mental, de los jóvenes y, por lo tanto, su desempeño académico y social. Es necesario, entonces, analizar la relación entre los estilos de vida y la salud en esta población. Así, el objetivo de esta investigación fue determinar la influencia del estilo de vida, el sexo, la edad y el índice de masa corporal sobre la salud física y psicológica de jóvenes universitarios, mediante una investigación explicativa con un diseño prospectivo único. Se usó una escala de estilos de vida, un Cuestionario de Salud General (GHQ-28), una escala de salud física y una hoja de identificación. Se evidenció que los 312 universitarios de la muestra, en general, gozan de buena salud. Reportan dificultades del sueño, gripe, dolor de huesos y cabeza. Presentan niveles bajos de depresión y malestar psicológico, con niveles moderados-bajos de ansiedad. Manifiestan hábitos inadecuados en alimentación, actividad física y chequeos médicos $y$, el consumo de drogas es bajo. Por medio de una regresión múltiple, se obtuvo que a mayor práctica de hábitos inadecuados de sueño y frecuencia de chequeos médicos mayores síntomas físicos. La mayor ansiedad se encuentra en mujeres, jóvenes con problemas de sueño y personas que consumen drogas. A menor edad y mayores problemas de sueño mayor depresión. El malestar psicológico se presenta con menor actividad física y problemas de sueño. Los resultados sugieren la conveniencia de crear programas de prevención en la universidad, tomando en cuenta los estilos de vida de los jóvenes.

Palabras clave: salud, estilos de vida, IMC, jóvenes universitarios.

* Profesora Asociada e investigadora en el Departamento de Ciencia y Tecnología del Comportamiento de la Universidad Simón Bolívar en Caracas, Venezuela. Investigadora del Centro de Investigación y Evaluación Institucional y Profesora Agregada de la Escuela de Psicología de la Universidad Católica Andrés Bello en Caracas, Venezuela. Correspondencia. Correo electrónico: angelucc@usb.ve

Cómo citar este artículo: Angelucci, L. T., Cañoto, Y., Hernández, M. J. (2017). Influencia del estilo de vida, el sexo, la edad y el IMC sobre la salud física y psicológica en jóvenes universitarios. Avances en Psicología Latinoamericana, 35(3), 531546. doi: http://dx.doi.org/10.12804/10.12804/revistas.urosario.edu.co/apl/a.4454 


\section{fibstract}

Changes in lifestyles that occur when entering college impact health, both physical and mental, therefore, the academic and social performance of young people. It is then necessary to evaluate the lifestyle-related health in this population. The aim of the research was to determine the influence of lifestyle, gender, age and body mass index on physical and psychological health of university students, through an explanatory research using a unique prospective design. The scales used were: Lifestyles Scale, General Health Questionnaire (GHQ-28), Physical Health Scale and an identification questionnaire. It was evident that the sample of 312 university students generally has good health. Reported sleep difficulties, flu, sore bones and head. They have low levels of depression and psychological distress, with moderate-low anxiety levels. Manifest inadequate diet, physical activity and health checks habits. Drug use is low. The multiple regression showed that inadequate sleep habits and low frequency of medical check-ups are associated with higher physical symptoms. Greater anxiety is present in women, young people with sleep problems and people who use drugs. The younger and those with sleep problems have major depression. The discomfort is associated with less physical activity and sleep problems. The results suggest the desirability of prevention programs at the university, considering the lifestyles of young people.

Keywords: Health, lifestyle, BMI, university students.

\section{Resumo}

As possíveis mudanças nos estilos de vida que se produzem ao entrar na universidade, em conjunto com outros fatores, impactam a saúde, tanto física quanto mental dos jovens e, portanto, o seu desempenho acadêmico e social; sendo necessário então analisar a relação estilos de vida- saúde nesta população. Assim, o objetivo da pesquisa foi determinar a influência do estilo de vida, o sexo, a idade e o índice de massa corporal sobre a saúde física e psicológica de jovens universitários, mediante uma investigação explicativa com um desenho prospetivo único. Empregou-se uma Escala de Estilos de Vidam um Questionário de Saúde Geral (GHQ-28), uma Escala de Saúde Física e uma folha de identificação. Evidenciou-se que os 312 universitários da amostra, em geral, gozam de boa saúde. Reportam dificuldades do sono, gripe, dor de ossos e cabeça. Apresentam níveis baixos de depressão e mal-estar psicológico, com níveis moderados-baixos de ansiedade. Manifestam hábitos inadequados em alimentação, atividade física e check-ups médicos e, o consumo de drogas é baixo. Por meio de uma regressão múltipla, obteve-se que com maior quantidade de hábitos inadequados de sono e frequência de check-ups médicos maiores sintomas físicos. Maior ansiedade encontra-se e mulheres jovens com problemas de sono e pessoas que consumem drogas. À menor idade e maiores problemas de sono, maior depressão. O mal-estar psicológico apresenta-se com menor atividade física e problemas de soo. Os resultados sugerem a conveniência de criar programas de prevenção na universidade, tomando em conta os estilos de vida dos jovens.

Palavras-chave: saúde, estilos de vida, IMC, jovens universitários.

\section{Introducción}

La población joven ha sido objeto de estudio de numerosas disciplinas. Sin embargo, este interés se "ha enfocado más en su problemática y menos en su normalidad y su salud" (Donoso, 1993, p. 1), lo que limita el campo de acción y la implantación de programas de prevención en salud para esta etapa de la vida.

La salud no se refiere únicamente a la ausencia de enfermedad. Varios autores, entre los que se citan Labiano (2002), la Organización Mundial de la Salud (OMS) (1946), Sebastiani, Pilicioni y Chiattone (2002), Seeman (1989) y Stone (1982), plantean que la salud puede ser entendida como bienestar físico, psicológico y social, y va más allá de lo puramente biomédico. Esta abarca lo subjetivo y el comportamiento. El individuo 
tiene un papel más activo, dado que puede prevenir determinadas enfermedades y fomentar o mantener el bienestar al realizar cambios comportamentales. Se destaca el papel preponderante de las variables conductuales y psicológicas en la determinación de la salud y la enfermedad (Angelucci, 2011).

La OMS, en su informe del 2008, da evidencia de que eso podría ser así, ya que ese año se produjeron 57 millones de defunciones a nivel mundial, de las cuales el $63 \%$ fueron el resultado enfermedades producidas por comportamientos o estilos de vida inadecuados (Angelucci, 2013). De esta manera, la prevención de la enfermedad y de accidentes de todo tipo, además de la promoción de la salud, deben ser temas de alta prioridad en las políticas y acciones de salud pública general, y sobre todo en la población productiva y potencialmente productiva, como la joven. Una de las propuestas para alcanzar este fin consiste en evaluar e intervenir los estilos de vida, como una de las acciones más eficaces (Sanabria, González \& Urrego, 2007).

El estilo de vida es definido por la OMS (1998) como "una forma general de vida basada en la interacción entre las condiciones de vida en un sentido amplio y los patrones individuales de conducta determinados por factores socioculturales y características personales" (p. 27). Se pueden entender como un conjunto de patrones conductuales o hábitos (Rodríguez-Marin \& García, 1995). Páez-Cala (2012) alude a la forma de vivir de las personas que se interrelaciona estrechamente con los componentes motivacionales y conductuales. En la adquisición y la consolidación de los estilos de vida, la adolescencia y juventud son etapas decisivas, puesto que los efectos de estas prácticas son multiplicativos, acumulativos y se observan a largo plazo (Morrison \& Bennet, 2008). En este sentido, las redes familiares y las instituciones educativas en las que se desenvuelven estos jóvenes tienen mucho que aportar (Páez-Cala \& Castaño-Castrillón, 2010).
Específicamente, para García-Laguna, García-Salamanca, Tapiero-Paipa y Ramos (2012), Pulido-Rull, Coronel-Villalobos, Vera-García y Barousse-Martínez (2011) y Schmidt (2012) el ingreso a la universidad conlleva cambios importantes en los hábitos, que tienen el potencial de afectar la salud psicológica y física, lo cual se manifiesta de muchas formas, por ejemplo en consumo de alcohol, tabaco, drogas, respuesta inapropiada al estrés, cambios desfavorables en la alimentación, experiencias sexuales riesgosas, menos actividad física, entre otros.

En la revisión de numerosos estudios realizados en estudiantes universitarios, en países tan distintos como España, India, Arabia Saudita, Inglaterra, Estados Unidos, Colombia, México, se muestra, en líneas generales, a un joven con un estilo de vida poco saludable, caracterizado por tener dificultades de sueño (Páez-Cala \& Castaño-Castrillón, 2010; Sierra, Jiménez \& Martin, 2002), baja actividad física (Deshpande, Patel, Bhujade \& Deepak, 2013; Gutiérrez et al., 2013; Khera \& Sharma, 2012; Varela-Mato, Cancela, Ayan, Martín \& Molina, 2012), consumo de drogas legales e ilegales, donde se destaca con mayor frecuencia el consumo de alcohol, cigarrillos y marihuana (Gutiérrez et al., 2013; Moreno-Gómez, et al., 2012; Varela-Mato, et al., 2012) y, finalmente, inadecuados hábitos alimentarios, representados por consumo de comida rápida, falta de desayuno, comer entre comidas y un alto consumo de carne, dulces, pastelería y gaseosas como bebida principal (Deshpande et al., 2013; El-Qudah, Al-Omran, Abu-Alsoud \& Al-Shek, 2012; Gutiérrez et al., 2013; Moreno-Gómez, et al., 2012; Vargas-Zarate, Becerra-Bulla \& Prieto-Suárez; 2008). En las investigaciones también se han encontrado diferencias en los estilos de vida entre hombres y mujeres, por ejemplo, los hombres reportan mayor presencia en actividades físico-deportivas, más actividades extracurriculares, mejor calidad de sueño y consumen menos dulces, no obstante, presentan más actividad sexual riesgosa, consumen 
más alcohol, cigarrillos y drogas ilegales, ingieren menos frutas y vegetales (El Ansarsi et al., 2011; Fountaine, Liguori, Mozumdar \& Schuna, 2011; Gutiérrez et al., 2013; Páez-Cala \& Castaño-Castrillón, 2010; Vargas-Zarate et al., 2008). Sin embargo, Varela-Mato et al. (2012) reportan que las mujeres consumían más alcohol y, según El-Qudah et al. (2012), tenían peores hábitos.

En cuanto a problemas de salud y enfermedades, el joven universitario se presenta como una persona sana, no obstante, se han detectado algunas enfermedades en un porcentaje importante. Así, por medidas antropométricas, se ha encontrado una presencia entre el $20 \%$ y el $45 \%$ de sobre peso u obesidad (Deshpande et al., 2013; Gutiérrez et al., 2013; Oviedo et al., 2008; Pulido-Rull et al., 2011; Rodríguez, Ríos, Lozano \& Álvarez, 2008; Vargas-Zarate et al., 2008), con mayor porcentaje de grasa corporal en las mujeres (Arrechabaleta, Castillo, Herrera \& Pacheco, 2002) y algunos estudiantes con peso por debajo de lo normal (El-Qudah et al., 2012). También se ha hallado hipertensión arterial (Arrechabaleta et al., 2002), ansiedad y depresión (Páez-Cala \& Cataño-Castrillón, 2010), migraña y hepatitis (Pulido-Rull et al., 2011; Rodríguez et al., 2008), amigdalitis, rinitis, insomnio y constipación (Martins-Bion, Castro-Chagas, Santana-Muniz \& Oliveira de Sousa, 2008), hiperlipidemia y alteraciones de los valores de glicemia en ayunas (Guerrero \& Machado, 2009), colitis, dolor de espalda crónico, úlcera gástrica y problemas bucales (Pulido-Rull et al., 2011). La salud se deteriora a medida que se prolonga el periodo universitario (Gutiérrez et al., 2013; Pulido-Rull et al., 2011) y hay mayores reportes de enfermedades en las mujeres (Pilgrim \& Blum, 2012).

Se evidencia así, que los jóvenes universitarios presentan inadecuados estilos de vida que pueden conllevar a problemas de salud, aunque manifiestan en la actualidad pocas enfermedades. Sin embargo, otros estudios indican que la relación entre el estilo de vida y la salud de los universitarios no es tan clara. Estudios al respecto, que se han enfocado más en la población adulta que en la adolescente, como los de Fernández-Ballesteros et al. (2010), García-Laguna et al. (2012) y Orejudo \& Froján (2005), muestran que las variables sociodemográficas son predictores más importantes de los síntomas de enfermedad que los estilos de vida, resaltándose el sexo, la edad, el nivel de estudio y el nivel de ingreso. De los estilos de vida, la actividad física y los hábitos alimentarios son los más relevantes (Esnaola, Infante, Rodríguez \& Goni, 2011; Remor \& Pérez-Llantada, 2007; Restrepo, Morales, Ramírez, López \& Varela, 2006). Otras investigaciones como las de Pardo-Torres y Núñez-Gómez (2008) y García-Laguna et al. (2012) agregan a la calidad de sueño, el fumar y el consumo de alcohol, como estilos de vida predictores de la salud.

En términos de la población universitaria, la información que se pueda aportar sobre los estilos de vida y la salud es muy relevante, ya que es un grupo de importancia social indiscutible que representa el capital humano en formación para el progreso de cualquier nación. En este sentido, el objetivo de la investigación es determinar la influencia del estilo de vida, el sexo, la edad y el IMC sobre la salud física y psicológica de estos jóvenes.

\section{Método}

\section{Participantes}

La muestra del estudio estuvo constituida por 312 estudiantes con diferentes años de estudio y carreras de una universidad privada de Caracas, Venezuela. La edad promedio fue 19,9 años. La mitad eran hombres. El IMC estuvo entre 14,8 y 45 , con un promedio de 23,04. El muestreo fue propositivo. El estudio fue aprobado por el respectivo Centro de Investigación al cual pertenecen las autoras. La participación de los estudiantes fue voluntaria, previo consentimiento informado. 


\section{Tipo y diseño de investigación}

La presente investigación es de tipo no experimental explicativo, debido a que no se tiene control directo sobre las variables y se pretende, a partir de un conjunto de variables, explicar el comportamiento de la salud de los jóvenes. Además, es un diseño de tipo transversal, ya que las respuestas de los sujetos se registran en un momento discreto en el tiempo (Kerlinger \& Lee, 2002). Según León y Montero (2003), es un diseño prospectivo de grupo único.

\section{Instrumentos}

Hoja de datos de identificación. En esta hoja se contemplaron datos reportados por el participante: sexo, edad, carrera, enfermedades físicas y discapacidades oculosensoriales.

Índice de masa corporal (IMC). El evaluador registraba la medida obtenida de peso y estatura, para obtener con estas el IMC. Para medir el peso en kilogramos se utilizó una báscula marca Tanita, modelo BF-679W7BF. Para la estatura en metros, se usó un tallímetro marca Saco.

Escala de salud física percibida. Escala construida por Angelucci (2011) para evaluar la salud física percibida por la frecuencia de síntomas físicos que se pueden relacionar con diferentes enfermedades. Se construyó sobre la base de cuestionarios de síntomas, entre los cuales se encuentra el de Tibblin, Bengtsson, Furunes \& Lapidus (1990), Krantz \& Östergren (1999), Jamal (2004) y Mellner, Krantz \& Lundberg (2005), los cuales presentan una adecuada confiabilidad (entre 0,79 y 0,93$)$ y validez de constructo. La escala está compuesta por 26 síntomas y se puntúa según la frecuencia de aparición del síntoma en las dos últimas semanas, según la escala — nunca (0) hasta muchas veces (3) - ; de modo que un puntaje mayor indica una peor salud. La confiabilidad de la escala es alta (alfa de Cronbach $=0,90)$ y su estructura unidimensional (Angelucci, 2011).
Cuestionario de salud general de Goldberg de 28 items (GHQ-28). Goldberg diseñó, en 1972, la escala de salud general (General Health Questionnaire, $\mathrm{GHQ}$ ) dirigida a evaluar la situación actual del sujeto con respecto al estado general de salud mental. En 1979, Goldberg y Hiller diseñaron un modelo de 28 ítems cuyas opciones de respuesta son presentadas por medio de una escala tipo Likert: "menos que lo habitual" (valor 0) hasta "más de lo habitual" (valor 3). Un mayor puntaje representa mayores problemas de salud. Esta escala ha sido ampliamente utilizada y validada en diferentes países anglosajones como Estados Unidos, de habla hispana como España, Colombia, Chile, Ecuador, entre otros y portuguesa como Brasil y Portugal. Para esta investigación los ítems se agruparon en tres factores, según estudios psicométricos previos en Venezuela: ansiedad y síntomas somáticos, depresión y falta de bienestar o disfunción social (Angelucci, 2011), donde además se presenta una alta consistencia interna (alpha de Cronbach =0,87).

Escala de estilo de vida saludable. Esta escala fue adaptada del cuestionario de prácticas y creencias sobre estilos de vida de Arrivillaga, Salazar \& Gómez (2002 citado en Arrivillaga, Salazar \& Correa, 2003; Salazar \& Arrivillaga, 2004), el cual se ha validado en países latinoamericanos como Colombia y México, con confiabilidades alrededor de 0,8. Está compuesto por 116 ítems con dos subescalas: prácticas (69 ítems) y creencias (47 ítems). Su formato de respuesta es de tipo Likert de cuatro alternativas con un rango de "siempre" a "nunca" y de "totalmente de acuerdo" a "totalmente en desacuerdo", respectivamente. El cuestionario evalúa siete dimensiones del estilo de vida: (i) condición, actividad física y deporte, (ii) tiempo de ocio, (iii) autocuidado y cuidado médico, (iv) hábitos alimentarios, (v) consumo de alcohol, (vi) tabaco y otras drogas y (vii) sueño. En esta investigación se usa solo la parte de prácticas, previa aplicación en muestras pilotos en estudiantes universitarios, con coeficiente alfas 
de Cronbach alrededor de 0,81 . Un mayor puntaje indica un estilo de vida saludable.

Para esta investigación las opciones de respuesta de las escalas se redujeron a dos (sí/no).

\section{Procedimiento}

El estudio se realizó siguiendo los lineamientos éticos para investigación en Psicología en uno de los laboratorios de la Escuela de Psicología de la UCAB - Caracas. Los participantes fueron estudiantes que se encontraban en el campus de la universidad y accedieron a participar. En primer lugar, se tomaban las medidas de peso y talla de cada joven y luego se les entregaban las escalas que debían responder de manera individual. Se les indicó un correo electrónico por si querían retroalimentación general de los resultados.

\section{Resultados}

En la muestra recogida para el presente estudio, el IMC promedio fue de 23. Al solicitar información sobre problemas sensoriales, motores y enfermedades crónicas, 124 (39,74\%) de los participantes reportaron usar lentes correctivos, dos utilizaban aparatos auditivos $(0,64 \%)$ y uno presentaba problemas motores $(0,32 \%)$. Ninguno de los participantes reportó presentar problemas de lenguaje. Un 14,42\% refirió padecer alguna enfermedad crónica no transmisible: asma $(9,24 \%)$, hipertensión (1,6\%), cardiopatía (1,28\%), epilepsia y úlcera $(0,64 \% \mathrm{c} / \mathrm{u})$, diabetes, hipotiroidismo y alergias con $0,32 \%$ cada uno.

En respuesta a la escala de síntomas físicos se encontró que los síntomas que son reportados por el $30 \%$ o más fueron: sueño durante el día, dolor de huesos, articulaciones o espalda, dolor de cabeza o jaqueca, tos, catarro o gripe y dificultad para dormir. Para los síntomas psicológicos, se obtuvo que los puntajes obtenidos en depresión fueron muy bajos, ningún síntoma de depresión se presentó en $30 \%$ o más de los participantes. Las preguntas más resaltantes para el factor ansiedad, indican que el $62 \%$ de los estudiantes manifiesta sentirse bien de salud y en plena forma, a pesar de que se reportan dolores de cabeza en un $64 \%$. Para la dimensión falta de bienestar psicológico, se tiene que más del $60 \%$ de los encuestados refiere que se mantiene activo, satisfecho, con la impresión de que está haciendo las cosas bien, disfruta de sus actividades, es capaz de tomar decisiones y desempeña un papel útil en la vida (ver tabla 1).

Tabla 1

Sintomas físicos de ansiedad y no bienestar más reportados por los jóvenes

\begin{tabular}{|c|c|c|}
\hline Síntomas Físicos & $\mathrm{N}$ & $\%$ \\
\hline Sueño durante el día & 179 & 57 \\
\hline Dolor de huesos, articulaciones o espalda & 166 & 53 \\
\hline Dolor de cabeza/jaqueca & 155 & 50 \\
\hline Tos, catarro o gripe & 119 & 38 \\
\hline Dificultad para dormir & 97 & 31 \\
\hline Síntomas Psicológicos (Ansiedad) & $\mathrm{N}$ & $\%$ \\
\hline $\begin{array}{l}\text { ¿Se ha sentido perfectamente bien de } \\
\text { salud y en plena forma? }\end{array}$ & 194 & 62 \\
\hline $\begin{array}{l}\text { ¿Se ha sentido agotado y sin fuerzas para } \\
\text { nada? }\end{array}$ & 95 & 30 \\
\hline $\begin{array}{l}\text { ¿Ha tenido la sensación de que estaba } \\
\text { enfermo? }\end{array}$ & 104 & 33 \\
\hline ¿Ha padecido dolores de cabeza? & 199 & 64 \\
\hline $\begin{array}{l}\text { ¿Sus preocupaciones le han hecho perder } \\
\text { mucho sueño? }\end{array}$ & 111 & 36 \\
\hline $\begin{array}{l}\text { ¿Ha tenido dificultades para dormir } \\
\text { seguido toda la noche? }\end{array}$ & 105 & 34 \\
\hline $\begin{array}{l}\text { ¿Se ha notado constantemente agobiado y } \\
\text { en tensión? }\end{array}$ & 105 & 34 \\
\hline $\begin{array}{l}\text { ¿Se ha sentido con los nervios "a flor de } \\
\text { piel" y malhumorado? }\end{array}$ & 99 & 32 \\
\hline $\begin{array}{l}\text { ¿Se ha notado nervioso y “a punto de } \\
\text { explotar"? }\end{array}$ & 93 & 30 \\
\hline Síntomas Psicológicos (No bienestar) & $\mathrm{N}$ & $\%$ \\
\hline $\begin{array}{l}\text { ¿Se las ha arreglado para mantenerse } \\
\text { ocupado y activo? }\end{array}$ & 195 & 63 \\
\hline
\end{tabular}


¿Ha tenido la impresión, en conjunto, de que está haciendo las cosas bien?

¿Se ha sentido satisfecho con su manera de hacer las cosas?

$218 \quad 70$

¿Ha sentido que está desempeñando un papel útil en la vida?

¿Se ha sentido capaz de tomar decisiones?

280

¿Ha sido capaz de disfrutar de sus actividades normales de cada día?

Fuente: elaboración propia.

Por otra parte, en la escala de estilos de vida, por medio de un análisis de componentes principales realizado en el presente estudio, se identificaron cinco factores: alimentación, actividad física, hábitos de sueño, uso de drogas y chequeos médicos. Al evaluar la respuesta en cada ítem, se obtuvo en la muestra hábitos inadecuados de alimentación, frecuencia de actividad física muy baja, así como pocos chequeos médicos, aunque son más activos al implementar otras conductas preventivas. Por otro lado, el reporte de los hábitos de sueño coincide con los reportados en la escala de síntomas físicos, donde se encontró que los estudiantes presentan diferentes problemas de sueño. El factor con mejores resultados es el de consumo de drogas, que tiene una frecuencia muy baja, aunque un $75 \%$ se automedica (tabla 2).

Tabla 2

Estilos de vida de salud más reportados por los jóvenes

\begin{tabular}{lc}
\hline \multicolumn{1}{c}{ Estilo de Vida } & $\%$ \\
\hline Factor 1: Alimentación & 86 \\
\hline Consume alimentos salados & 84 \\
Come en forma balanceada & 73 \\
Consumo de dulces & 81 \\
Consumo de golosinas o chucherías & 80 \\
Consumo de embutidos & 73 \\
Consumo de comidas con colorantes y \\
preservantes
\end{tabular}

Factor 2: Ejercicio físico

Practica ejercicios de relajación 17

Participa en actividades físicas programadas $\quad 24$

Incluye descansos en su rutina $\quad 14$

Realiza actividades de recreación $\quad 6$

Factor 3: Sueño

Trasnocho 30

Incluye momentos de descanso 86

Duerme al menos 7 horas 62

Se trasnocha 30

Se levanta descansado 53

Le cuesta quedarse dormido 67

Se despierta varias veces en la noche $\quad 67$

Hace siesta 39

Se mantiene con sueño parte del día

Utiliza pastillas para dormir $\quad 96$

Factor 4: Uso de drogas

Automedicación de trastornos leves (gripe, dolores de cabeza)

Lee las instrucciones de los medicamentos 24

Consumo de ansiolíticos o estimulantes sin 5 prescripción médica

Consumo de alcohol 25

Consumo de café 22

Fuma 13

Consumo de drogas $\quad 7$

Consumo de pastillas para dormir 4

Factor 5: Chequeo médico

Chequeo de la presión arterial 28

Chequeo médico $\quad 17$

Exámenes médicos 32

Fuente: elaboración propia.

Ahora bien, con el objetivo de analizar en qué medida los estilos de vida, el sexo, la edad y el IMC influyen la salud física y psicológica de los jóvenes, se realizaron cuatro regresiones múltiples 
(una para cada dimensión de salud). Previamente, se verificaron los supuestos de la regresión lineal (adecuación de los errores, no multicolinealidad, en la tabla 3 se pueden observar las correlaciones entre las variables), la normalidad, a excepción de síntomas físicos, depresión y falta de bienestar, donde se hicieron algunas trasformaciones, pero no se ajustaron. Se procedió al análisis de regresión, considerándose que esta asimetría se da por las características de la muestra y lo que es medido por las escalas.

Como se puede ver en la tabla 4 , los resultados de la regresión múltiple indican que a mayor estilo inadecuado en sueño y mayor frecuencia de chequeo médico mayores síntomas físicos. El presentar problemas de sueño, el ser mujer y el tener un mayor consumo de drogas se asocia con una mayor ansiedad. Tener problemas de sueño y poseer menor edad lleva a una mayor depresión, y, por último, poseer problemas de sueño y el tener baja actividad física conlleva a poco bienestar. El IMC y los hábitos alimentarios no explican alguna de las dimensiones de salud evaluadas. La ansiedad fue la dimensión más explicada.

\section{Discusión}

Primeramente, la muestra evaluada presentó un IMC de 23, clasificándose a los estudiantes como normales; aunque, al analizar los extremos, parece ser mayor el exceso de peso que su déficit. Estos resultados son similares a los de Córdoba et al. (2013), Deshpande et al. (2013), Gutiérrez et al. (2013) y Lara et al. (2012), entre otros. Se encontró que el IMC no se relacionó con el sexo, contrario a lo hallado por Arechavaleta et al. (2002).

Tabla 3

Matriz correlaciones entre las variables

\begin{tabular}{|c|c|c|c|c|c|c|c|c|c|c|c|c|}
\hline & $\begin{array}{l}\text { Síntomas } \\
\text { Físicos }\end{array}$ & Ansiedad & $\begin{array}{l}\text { Depre- } \\
\text { sión }\end{array}$ & $\begin{array}{c}\text { No } \\
\text { bienestar }\end{array}$ & $\begin{array}{l}\text { Alimen- } \\
\text { tación } \\
\text { balan- } \\
\text { ceada }\end{array}$ & Ejercicio & Sueño & $\begin{array}{c}\text { No } \\
\text { drogas }\end{array}$ & $\begin{array}{l}\text { Chequeo } \\
\text { médico }\end{array}$ & $\begin{array}{c}\text { Índice } \\
\text { de masa } \\
\text { corporal }\end{array}$ & Edad & Sexo \\
\hline $\begin{array}{l}\text { Síntomas } \\
\text { físicos }\end{array}$ & 1 & $0,268 * *$ & 0,07 & $0,117^{*}$ & $-0,031$ & $-0,057$ & $-0,241 * *$ & $-0,083$ & $0,149 * *$ & 0,037 & $-0,028$ & $0,190 * *$ \\
\hline Ansiedad & & 1 & $0,337 * *$ & $0,305^{* *}$ & 0,029 & $-0,191 * *$ & $-0,494 * *$ & $-0,189 * *$ & $-0,012$ & 0,084 & $-0,03$ & $0,304 * *$ \\
\hline Depresión & & & 1 & $0,317 * *$ & 0,051 & $-0,032$ & $-0,207 * *$ & $-0,084$ & $-0,057$ & 0,003 & $-0,088$ & 0,059 \\
\hline No bienestar & & & & 1 & $-0,02$ & $-0,186^{* *}$ & $-0,305^{* *}$ & $-.0,107$ & $-0,156^{* *}$ & 0,061 & $-0,008$ & 0,063 \\
\hline $\begin{array}{l}\text { Alimentación } \\
\text { balanceada }\end{array}$ & & & & & 1 & $-0,052$ & 0,046 & 0,077 & $0,185^{* *}$ & 0,055 & 0,019 & $0,179 * *$ \\
\hline Ejercicio & & & & & & 1 & $0,195 * *$ & $-0,005$ & 0,007 & $-0,029$ & 0,071 & $-0,269 * *$ \\
\hline Sueño & & & & & & & 1 & 0,026 & 0,081 & $-0,140 *$ & $-0,049$ & $-0,252 * *$ \\
\hline No drogas & & & & & & & & 1 & $0,120^{*}$ & $-0,037$ & $-0,164 * *$ & 0,043 \\
\hline $\begin{array}{l}\text { Chequeo } \\
\text { médico }\end{array}$ & & & & & & & & & 1 & 0,078 & $-0,057$ & $0,300 * *$ \\
\hline $\begin{array}{l}\text { Índice de ma- } \\
\text { sa corporal }\end{array}$ & & & & & & & & & & 1 & $0,196^{* *}$ & $\quad-0,11$ \\
\hline Edad & & & & & & & & & & & 1 & $-0,029$ \\
\hline Sexo & & & & & & & & & & & & 1 \\
\hline
\end{tabular}

*Significativo al $5 \%$.**Significativo al 1

Fuente: elaboración propia. 
Tabla 4

Coeficientes de regresión para cada una de las dimensiones de salud

\begin{tabular}{|c|c|c|c|c|c|c|c|c|c|c|c|c|}
\hline \multirow{2}{*}{$\begin{array}{c}\text { Variables predic- } \\
\text { toras }\end{array}$} & \multicolumn{3}{|c|}{ Síntomas físicos } & \multicolumn{3}{|c|}{ Ansiedad } & \multicolumn{3}{|c|}{ Depresión } & \multicolumn{3}{|c|}{ No bienestar } \\
\hline & Beta & $\mathrm{t}$ & Sig & Beta & $\mathrm{t}$ & Sig & Beta & $\mathrm{t}$ & Sig & Beta & $\mathrm{t}$ & Sig \\
\hline Constante & & 2,098 & 0,037 & & 4,835 & 0,00 & & 3,217 & 0,001 & & 3,637 & 0,00 \\
\hline Sexo & 0,109 & 1,675 & 0,95 & 0,213 & 3,729 & $0,00 * *$ & 0,19 & 0,284 & 0,777 & 0,006 & 0,102 & 0,919 \\
\hline Edad & $-0,031$ & $-0,538$ & 0,591 & $-0,074$ & -1.459 & 0,146 & $-0,118$ & $-2,027$ & $0,044^{*}$ & $-0,053$ & $-0,943$ & 0,346 \\
\hline $\begin{array}{l}\text { Índice de masa } \\
\text { corporal }\end{array}$ & 0,023 & 0,387 & 0,699 & 0,053 & 1,02 & 0,309 & $-0,005$ & $-0,079$ & 0,937 & 0,18 & 0,316 & 0,752 \\
\hline $\begin{array}{l}\text { Alimentación } \\
\text { balanceada }\end{array}$ & $-0,063$ & $-1,096$ & 0,274 & 0,026 & 0,514 & 0,607 & 0,082 & 1,411 & 0,159 & $-0,013$ & $-0,225$ & 0,822 \\
\hline Ejercicio & 0,023 & 0,399 & 0,69 & $-0,05$ & -0.959 & 0,338 & 0,029 & 0,491 & 0,624 & $-0,121$ & $-2,075$ & $0,039^{*}$ \\
\hline Sueño & $-0,22$ & $-3,664$ & $0,00 * *$ & $-0,419$ & $-7,933$ & $0,00 * *$ & $-0,225$ & $-3,703$ & $0,00 * *$ & $-0,277$ & $-4,683$ & $0,00 * *$ \\
\hline No drogas & $-0,106$ & $-1,856$ & 0,064 & $-0,197$ & $-3,929$ & $0,00 * *$ & $-0,105$ & $-1,821$ & $0,07 *$ & $-0,95$ & $-1,704$ & 0,089 \\
\hline Chequeo médico & 0,138 & 2,247 & $0,025^{*}$ & $-0,026$ & $-0,475$ & 0,635 & $-0,033$ & $-0,528$ & 0,598 & $-0,107$ & $-1,782$ & 0,076 \\
\hline
\end{tabular}

*Significativo al $5 \%$. * Significativo al $1 \%$

Fuente: elaboración propia.

En cuanto a las discapacidades reportadas, el uso de lentes correctivos en un $39,4 \%$ coincide con los resultados de Garrido (2011) en estudiantes madrileños. Para las enfermedades padecidas por la muestra, se encontró una mayor prevalencia de asma, luego hipertensión y, por último, cardiopatía. El porcentaje de estudiantes que indica enfermedades es muy bajo si se compara con reportes de otros estudios (Martins-Bion et al., 2008; Oviedo et al., 2007; Pulido-Rull et al., 2011), tal vez porque en esos estudios se declaran como enfermedades aspectos que en la presente investigación son evaluados como síntomas. Por otra parte, el $33 \%$ de los jóvenes manifestó sentirse enfermo. Esta percepción parece estar relacionada con un conjunto de síntomas físicos que presentan alrededor de la mitad de los jóvenes evaluados, asociados con alteraciones del sueño, dolores de huesos, dolores de cabeza y tos o gripe. De hecho, algunos estudiantes de la muestra dicen padecer amigdalitis, rinitis, insomnio, constipación, diabetes, dolores de espalda, úlceras, migraña y problemas bucales, al igual que en los estudios de Martins-Bion et al. (2008) y Pulido-Rull et al. (2011).

Al analizar los resultados sobre la salud psicológica, los estudiantes no refieren síntomas relacionados con depresión. El no encontrar depresión no coincide con lo que otros investigadores señalan (Páez-Cala \& Castaño-Castrillón, 2010), esto puede deberse a diferencias en la evaluación, ya que en la presente investigación se usó la escala de Golberg, la cual mide depresión grave y, tal como se espera en una población sana, los síntomas fueron bajos. Los alumnos reportan sentirse bien y desempeñando un papel útil en la vida, capaces de tomar decisiones y disfrutar de las actividades cada día. Este elemento es muy alentador, ya que se trata de una muestra de la población joven y en preparación para colaborar con el desarrollo del país. Lo anterior se ajusta a lo encontrado en muestras de sujetos no hospitalizados (Escobar \& Pico, 2013), integrantes de una comunidad universitaria (González \& Landero, 2008) y en jóvenes universitarios venezolanos (Angelucci, 2011; Pacheco, Suárez \& Angelucci, 1998). 
El hecho de que los estudiantes presenten adecuada salud puede ser explicado por las características de la muestra: estudiantes de una institución privada, con nivel socioeconómico medio, elementos que en otras investigaciones se ha asociado con una buena salud (Gallo \& Matthews, 2003; Kielbasiewicz-Drozdowska, 2007; Taylor, Lerner, Sage, Lehman \& Seeman, 2004). Sin embargo, manifiestan ansiedad moderada, mencionando dificultades para dormir, dolor de cabeza, sentirse agobiados y en tensión, malhumorados y agotados. Esta ansiedad se puede asociar con el mismo contexto universitario (demandas académicas) y con las responsabilidades y preocupaciones de este periodo etario.

En cuanto a los estilos de vida, los jóvenes reportaron problemas de sueño, hábitos inadecuados de alimentación, actividad física muy baja, pocos chequeos médicos, aunque bajo consumo de drogas. Estos datos coinciden con investigaciones en poblaciones similares (Córdoba et al., 2013; Gutiérrez et al., 2013; Martins-Bion et al., 2012; Pérez-Cala \& Castaño-Castrillón, 2010; Sierra et al. 2002; Solórzano, 2013). Aunque no en relación al dato de chequeo médico (Escobar \& Pico, 2013). El poco tiempo del que disponen los estudiantes para las responsabilidades académicas puede explicar las altas tasas de sedentarismo, unido al acceso a tecnologías y ocio pasivo, así como a la disponibilidad de transporte (el metro y la parada de autobús quedan frente a la universidad). El abuso de golosinas, la poca accesibilidad en la universidad a alimentos sanos (no existe comedor), la propaganda constante de consumo de alimentos refinados, la influencia de pares y la escasez de alimentos regulados podrían explicar la alimentación inadecuada. Por su parte, los problemas de sueño pueden deberse a las responsabilidades académicas (se acuestan muy tarde haciendo tareas) o entretenimiento por medio de internet o televisión a altas horas de la noche. Se infiere que los inadecuados hábitos de salud están relacionados unos con otros y no funcionan de manera aislada, como en el presente caso.

Ahora bien, al evaluar cómo estos estilos de vida, junto con el sexo y el IMC influyen en la salud de estos jóvenes, se obtuvo que las dimensiones de salud fueron influidas por algunas de las variables, pero con poco porcentaje de varianza explicada. Este hallazgo concuerda con lo establecido por Orejudo \& Froján (2005), al indicar que las variables sociodemográficas y los estilos de vida no son los predictores más importantes de los síntomas de enfermedad. Sin embargo, entre los estilos de vida que contribuyeron a la explicación de la salud de los jóvenes, se obtuvo que el sueño fue el más importante, dado que predice todos los indicadores de salud. Los jóvenes que presentaron hábitos inadecuados de sueño reportaron peor salud física, mayor ansiedad, depresión y poco bienestar. La importancia del sueño ya ha sido recalcada por diferentes investigadores en su relación con la salud (Páez-Cala \& Castaño-Castrillón, 2010; Pardo-Torres \& Núñez-Gómez, 2008; Sierra et al.2002) y debe tomarse en cuenta para posibles programas de prevención, considerando además, que es uno de los estilos de vida inadecuados en estos jóvenes. No realizar actividad física también predijo la salud, específicamente el bajo bienestar. El sedentarismo ha sido asociado con la salud y, en mayor cuantía, con los aspectos relacionados con el estrés, sentimiento de soledad y poco disfrute del tiempo libre (Esnaola et al., 2011; Fernández-Ballesteros et al., 2010; Pardo-Torres \& Núñez-Gómez, 2008), aspectos relacionados con la medida de no bienestar de la presente investigación; sin embargo, también se ha visto que afecta los síntomas de malestar físico, la ansiedad y la depresión (Martins-Bion et al., 2008; Remor \& Pérez-Llantada, 2007), lo que no se halló en el presente estudio, tal vez porque los jóvenes de esta muestra asocian la actividad física con el entretenimiento, interacción social y manejo del ocio y, por ende, su bienestar psicológico. 
Por otra parte, el consumo de drogas predijo una mayor ansiedad y el chequeo médico una peor salud física. Estas relaciones pueden considerarse bidireccionales, en tanto el uso continuado de una sustancia puede conducir a experimentar ansiedad o algunos trastornos de ansiedad pueden cursar con un mayor consumo de ciertas drogas. Así mismo, una salud física deteriorada puede estimular los chequeos médicos, y a la vez los resultados de los chequeos médicos conllevan una mayor atención a los síntomas de enfermedad. Por último, los factores sociodemográficos (edad y sexo) influenciaron los niveles de salud de los jóvenes, la depresión y la ansiedad, respectivamente, donde hay mayor depresión a menor edad y mayor ansiedad en las mujeres. Las investigaciones señalan que a mayor edad, mayor es el riesgo de padecer enfermedades (Fernández-Ballesteros et al., 2010); sin embargo, la muestra más joven de este estudio parece estar más vulnerable a los estímulos negativos que causan tristeza y melancolía. El hecho de que las mujeres reporten mayor ansiedad, concuerda con estudios previos (Cauffman, Lexcen, Goldweber, Shulman \& Grisso, 2007; Matud \& Aguilera, 2009; Nolen-Hoeksema \& Girgus, 1994). Aunque no se encontraron, tal como dice la literatura (Afifi, 2006; Maciejewski, Prigerson \& Mazure, 2001), mayores síntomas físicos y mayor depresión, sino solo mayor ansiedad.

Existen varias explicaciones al por qué las mujeres reportan más síntomas. Nolen-Hoeksema \& Girgus (1994) establecen que las mujeres prestan mayor atención hacia su cuerpo. Otra explicación se enfoca en los estilos de vida saludables. Se ha reportado que los hombres presentan más conductas de riesgo de salud que las mujeres, al considerar casi todas las conductas saludables (Córdoba et al., 2013; Páez-Cala \& Castaño-Castrillón, 2010; Pilgrim \& Blum, 2012; Pulido-Rull et al., 2011), lo que se evidenció en esta investigación. Sin embargo, los hombres evalúan mejor su estatus de salud general, estableciéndose explicación la poca vulnerabilidad percibida a la enfermedad que presentan (Dawson et al., 2007). Por otra parte, es posible que las jóvenes manifiesten más estrés académico en el contexto universitario (Román, Ortiz \& Hernández, 2008; Rosario et al., 2008).

Esta investigación pone de manifiesto, como muchas otras, que los estilos de vida en jóvenes no son tan saludables y que, a pesar de que estos jóvenes gozan, en líneas generales, de buena salud en el presente, manifiestan un conjunto de conductas que pueden influir negativamente a futuro en su salud física y psicológica. Dada la importancia de estas conductas con respecto al desarrollo de enfermedades crónicas o la presencia de síntomas físicos que interfieren con el buen funcionamiento general, y específicamente el desempeño académico, sería recomendable intervenir en niveles (Escobar \& Pico, 2013), y la universidad puede convertirse en una universidad saludable (Páez-Cala, 2012). En este sentido, García-Laguna et al. (2012) señalan estrategias, tales como: (i) implementar materias obligatorias y electivas, referidas a la práctica estilos de vida saludable, (ii) crear espacios para la participación en actividades físicas, (iii) mejorar el acceso a alimentos saludables, (iv) generar campañas y regulaciones para el no consumo de drogas.

Se debe tomar en cuenta que la robustez de estas conclusiones se ve limitada por el uso de medidas de auto-informe, así mismo el modelo propuesto es parcial y explica solo una parte de la salud bajo ciertas especificaciones conceptuales. Como señalan Nunes-Baptista, García-Capitão \& A1ba-Scortegagna (2006), la salud está determinada por variables etiológicas, genéticas, bioquímicas, conductuales y socio-ambientales. Esta gran cantidad de variables se relacionan de modo complejo, lo que hace que la comprensión de este fenómeno también lo sea.

Así, se propone seguir investigando en poblaciones universitarias, tomando en cuenta otros factores relacionados con la salud y los estilos de vida, lo que permitiría aumentar la comprensión 
del fenómeno salud y, en consecuencia, redundaría en políticas de prevención.

\section{Referencias}

Almagia, F., Lizana, A., Rodríguez, R., Ivanovic, D., \& Binvignat, G. (2009). Variables antropométricas y rendimiento físico en estudiantes universitarios de educación física. International Journal of Morphology, 27(4), 971-975. doi: http://dx. doi.org/10.4067/S0717-95022009000400001

Afifi, M. (2006). Positive health practices and depressive symptoms among high school adolescents in Oman. Singapore Medicine Journal, 47, 960-966. Recuperado de http://www.sma. org.sg/smj/4711/4711a8.pdf

Angelucci, L. (2011). Emoción y salud: Un modelo causal. (Tesis inédita de doctorado, Universidad Central de Venezuela, Caracas, Venezuela).

Angelucci, L. (2013). Hacia un vivir saludable. Debates IESA, 18(2), 16-20.

Arechabaleta, G., Castillo, H., Herrera, H., \& Pacheco, M. (2002). Composición corporal en una muestra de estudiantes universitarios. Revista de la Facultad de Medicina, UCV, 25(2), 209-216. Recuperado de http://www. scielo.org.ve/scielo.php?script=sci_arttext\&pi$\mathrm{d}=$ S0798-04692002000200009\&lng=es\&nr$\mathrm{m}=$ iso

Arrivillaga, M. Salazar, I., \& Correa, D. (2003). Creencias sobre la salud y su relación con las prácticas de riesgo o de protección en jóvenes universitarios. Colombia Médica, 34(4), 187195. Recuperado de http://www.redalyc.org/ articulo.oa? $\mathrm{id}=28334403$

Berdasco, A. (2002). Evaluación del estado nutricional del adulto mediante la antropometría. Revista Cubana de Alimentación y Nutrición, 16, 146-152. Recuperado de http://www.bvs. sld.cu/revistas/ali/vol16_2_02/ali09202.pdf

Cauffman, E., Lexcen, F., Goldweber, A., Shulman, E., \& Grisso, T. (2007). Gender differences in mental health symptoms among delinquent and community youth. Youth Violence and Juvenile Justice, 5, 287-307. Recuperado de http://yvj. sagepub.com/content $/ 5 / 3 / 287$.short

Córdoba, D., Carmona, M., Terán, O., \& Márquez, O. (2013). Life style and nutritional status in university students: A descriptive, cross-sectional study. Medwave, 13(11). doi: 10.5867/ medwave.2013.11.5864

Dawson, K., Schneider, M., Fletcher, P., \& Bryden, P. (2007). Examining gender differences in the health behaviors of Canadian university students. The Journal of the Royal Society for the Promotion of Health, 127, 38-44. Recuperado de http://jmh.sagepub.com/content/5/5/413. full.pdf + html

Deshpande, K., Patel, S., Bhujade, R., \& Deepak, P. (2013). Lifestyle and obesity among college students in Ujjain, India. National Journal of Community Medicine, 4(2), 291-293. Recuperado de http://njcmindia.org/uploads/4-2_291-293.pdf Donoso, L. (1993). Salud del adolescente. Avances en Enfermería, 11(1), 29-36. Recuperado de http://www.bdigital.unal.edu.co/20468/1/1659851882-1-PB.pdf

El Ansari, W., Stock, Ch., John, J., Deeny, P., Phillips, C., Snelgrove, S., et al. (2011). Health promoting behaviours and lifestyle characteristics of students at seven universities in the UK. European Journal of Public Health, 19(4), 197-204. Recuperado de http://apps.szu.cz/svi/ cejph/archiv/2011-4-03-full.pdf

El-Qudah, J., Al-Omran, H., Abu-Alsoud, B. \& AlShek, T. (2012). Nutritional status among a simple of saudi college students. Current Research Journal of Biological Sciences, 4(5), 557-562. Recuperado de http://maxwellsci.com/print/ crjbs/v4-557-562.pdf

Escobar, M., \& Pico, M. (2013). Autocuidado de la salud en jóvenes universitarios. Revista Facultad Nacional de Salud Pública, 31(2), 178186. Recuperado de http://www.redalyc.org/ pdf/120/12028113003.pdf 
Esnaola, I., Infante, G., Rodríguez, A., \& Goñi, E. (2011). Relación entre las variables psicosociales y salud percibida. Revista de Psicología del Deporte, 20(2), 413-427. Recuperado de www. rpd-online.com/article/download/1003/780

Espinosa, L. (2004). Cambios del modo y estilo de vida: Su influencia en el proceso salud-enfermedad. Revista Cubana de Estomatología, 41(3). Recuperado de http://scielo.sld.cu/scielo.php?scrip$\mathrm{t}=$ sci_arttext\&pid=S0034-75072004000300009

Fernández-Ballesteros, R., Zamarrón, M. D., López Bravo, M. D., Molina, M. A., Díez, J., Montero, P., \& Schettini, R. (2010). Envejecimiento con éxito: Criterios y predictores. Psicothema, 22(4), 641-647. Recuperado de http://digital. csic.es/bitstream/10261/83676/1/Envejecimiento $\% 20$ con $\% 20 \% \mathrm{C} 3 \%$ A 9 xito $\% 20$ criterios $\% 20$ y\%20predictores.pdf

Fountaine, Ch., Liguori, G., Mozumdar, A., \& Schuna, J. (2011). Physical activity and screen time sedentary behaviours in college students. International Journal of Exercise Science, 4(2), 102-112. Recuperado de http://digitalcommons. wku.edu/cgi/viewcontent.cgi?article $=1279 \&$ context $=$ ijes

Gallo, L., \& Matthews, K. (2003). Understanding the association between socioeconomic status and physical Health: Do negative emotions play a role? Psychological Bulletin, 129(1), 10-51. Recuperado de http://www.sci.sdsu.edu/ lcgallo/gallopb.pdf

García-Laguna, D., García-Salamanca, G., Tapiero-Paipa, Y., \& Ramos, D. (2012). Determinantes de los estilos de vida y su implicación en la salud de jóvenes universitarios. Revista Hacia la Promoción de la Salud, 17(2), 169185. Recuperado de http://www.redalyc.org/ pdf/3091/309126826012.pdf

Garrido, R. (2011). Epidemiología descriptiva del estado refractivo en estudiantes universitarios. (Tesis inédita de doctorado, Universidad Complutense de Madrid, Madrid, España).
González-Ramírez, M., Landero-Hernández, R., \& Ruiz-Díaz, M. A. (2008). Modelo estructural predictor de la salud mental y física en mujeres. Revista Panamericana de Salud Pública, 23(2), 101-108. Recuperado de http://www.scielosp. org/pdf/rpsp/v23n2/05.pdf

Guerrero C., \& Machado J. (2009). Factores de riesgo de enfermedades crónicas en estudiantes universitarios. Ciencia y Salud, 11(2), 11-20. Recuperado de http://revistas.curnvirtual.edu. co/index.php/cienciaysalud/article/view/60

Gutiérrez, G., Meaney, A., Ocharan, M., Araujo, J., Ramírez, I, Olivares-Corichi. I., et al. (2013). Anthropometric traits, blood pressure, and dietary and physical exercise habits in health sciences students: The Obesity Observatory Project. Nutrición Hospitalaria, 28(1), 194-201. doi: 10.3305/nh.2013.28.1.6185

Hernández V., Riveros, Q., \& Rivera B. (2007). Niveles de depresión y ansiedad en estudiantes universitarios de Lima metropolitana. Revista de Investigación en Psicología, 10(1), 91-102. Recuperado de https://dialnet.unirioja.es/servlet/articulo;jsessionid $=05 \mathrm{D} 1 \mathrm{BC}$ 230B3041ACE9084B7EBDB89C87.dialnet02? codigo $=2348649$

Jamal, M. (2004). Burnout, stress and health of employees on non-standard work schedules: a study of Canadian workers. Stress and Health, 20, 113-119. Recuperado de http://www.ingentaconnect.com/content/jws/ smi/2004/00000020/00000003/art00003

Khera, R., \& Sharma, R. (2012). Physical inactivity among college students in associated with living in hostels. Global Journal of Medicine and Public Health, 1(5), 82- 85. Recuperado de https:// www.researchgate.net/publication/289122551 Physical_inactivity_among_college_students is_associated_with_living_in_hostels_a study_ from_Delhi_India

Kiełbasiewicz-Drozdowska, I. (2007). Students' pro-healthy lifestyle choices - social and economic determinants. Research Yearbook, 13(2), 
254-258. Recuperado de http://webcache. googleusercontent.com/search?q=cache:oiDR31MuqOUJ:jm12012.indexcopernicus. com/fulltxt.php\%3FICID\%3D982908+\&c$\mathrm{d}=2 \& \mathrm{hl}=\mathrm{es} \& \mathrm{ct}=\mathrm{clnk} \& \mathrm{gl}=\mathrm{ve}$

Krantz, G., \& Östergren, P. (1999). Women's health: do common symptoms in women mirror general distress or specific disease entities? Scandinavian Journal of Public Health, 27, 311-317. Recuperado de http://sjp.sagepub. com/content $/ 27 / 4 / 311$.short

Labiano, L. (2002). Hipertensión arterial: Importancia de las intervenciones psicológicas. Psicología Iberoamericana, 10(2), 64-71. Recuperado de http://www.redalyc.org/pdf/180/18011328004. pdf

Lara, R., García, J., Zúñiga, M., \& Parra, J. (2012). La autopercepción corporal y el IMC como indicadores del estado nutricional de estudiantes universitarios. Nutrición Clínica y Dietética Hospitalaria, 32(2), 86-91. Recuperado de http://www.nutricion.org/publicaciones/revista_2012_32_2/AUTOPERCEPCION-CORPORAL.pdf

León, O., \& Montero, I. (2003). Métodos de Investigación en psicología y educación (3. ${ }^{a}$ edición). Madrid: McGraw-Hill.

Maciejewski, P., Prigerson, H., \& Mazure, C. (2001). Sex differences in event-related risk for major depression. Psychological Medicine, 31, 593-604. doi: http://dx.doi.org/10.1017/ S0033291701003877

Martins-Bion, F., Castro-Chagas, M., de Santana-Muniz, G., \& Oliveira-de Sousa, L. (2008). Estado nutricional, medidas antropométricas, nivel socioeconómico y actividad física en universitarios brasileños. Nutrición Hospitalaria, 23(3), 234241. Recuperado de http://scielo.isciii.es/scielo. php?pid=S0212-16112008000300010\&scrip$\mathrm{t}=$ sci_arttext

Matud, M., \& Aguilera, L. (2009). Roles sexuales y salud mental en una muestra de la población general española. Salud Mental, 32,
53-58. Recuperado de http://www.scielo. org.mx/scielo.php?script=sci_arttext\&pi$\mathrm{d}=\mathrm{S} 0185-33252009000100007$

Mellner, C., Krantz, G., \& Lundberg, U. (2005). Medically unexplained symptoms in women as related to physiological stress responses. Stress and Health, 21, 45-52. doi: 10.1002/smi.1037

Moreno-Gómez, C., Romaguera-Bosch, D., Tauler-Riera, P., Bennasar-Veny, M., Pericas-Beltran, J., Martinez-Andreu, S., et al. (2012). Clustering of lifestyle factors in spanish university students: relationship between smoking, alcohol consumption, physical activity and diet quality. Public Health Nutrition, 15(11), 2131-2139. doi: http://dx.doi.org/10.1017/ S1368980012000080

Morrison, V., \& Bennet, P. (2008). Psicología de la salud. Madrid: Pearson.

Nolen-Hoeksema, S., \& Girgus, J. (1994). The emergent of gender differences in depression during adolescence. Psychological Bulletin, 115, 424443. doi: 10.1037/0033-2909.115.3.424

Nunes-Baptista, M., Garcia-Capitão, C., \& Alba-Scortegagna, S. (2006). Evaluación psicológica en la salud: Contextos actuales. Estudios Sociales, 15(28), 138-161. Recuperado de http://www. redalyc.org/articulo.oa? $\mathrm{id}=41702805$

Orejudo, O., \& Froján, X. (2005). Síntomas somáticos: Predicción diferencial a través de variables psicológicas, sociodemográficas, estilos de vida y enfermedades. Anales de Psicología, 21(2), 276-285. Recuperado de http://revistas.um.es/ analesps/article/viewFile/26871/26071

Organización Mundial de la Salud (1946). ¿Cómo define la oms la salud? Recuperado de http:// www.who.int/whr/2008/es

Organización Mundial de la Salud (2008). Informe sobre la salud en el mundo. Recuperado de http://www.who.int/whr/2008/es

Organización Mundial de la Salud (2012). Estadisticas sanitarias mundiales. Recuperado de http:// www.who.int/whr/2008/es 
Oviedo, G., Morón, A., Santos, I., Sequera, S., Soufrontt, G., Suárez, P., et al. (2008). Factores de riesgo de enfermedades crónicas no transmisibles en estudiantes de la carrera de medicina, Universidad de Carabobo, Venezuela. Año 2006. Nutrición Hospitalaria, 23(3), 288-293. Recuperado de http://scielo.isciii.es/pdf/nh/ v23n3/original13.pdf

Pacheco, A., Suárez, L., \& Angelucci, L. (1998). Diagrama de ruta del efecto de los factores sociodemográficos, depresión y apoyo social sobre la salud percibida en estudiantes universitarios. Suma Psicológica, 5, 149-191. Recuperado de http://publicaciones.konradlorenz.edu.co/index. $\mathrm{php} /$ sumapsi/article/view/254

Páez-Cala, M., \& Castaño-Castrillón, J. (2010). Estilos de vida y salud en estudiantes de una facultad de psicología. Psicología desde el Caribe, 25, 155-178. Recuperado de http://www.redalyc. org/articulo.oa? $\mathrm{id}=21315106008$

Páez-Cala, M. (2012). Universidades saludables: Los jóvenes y la salud. Archivos de Medicina, 12(2), 205-220. Recuperado de http://www.redalyc. org/articulo.oa? id=273825390007

Pardo, M., \& Torres, N. (2008). Estilo de vida y salud en la mujer adulta joven. Aquichan, 8(2), 266-284. Recuperado de http://www.redalyc. org/articulo.oa? id=74180212

Piédrola, G. (2002). Medicina preventiva y salud pública. Barcelona, España: Masson, S. A.

Pilgrim, N., \& Blum, R. (2012). Adolescent mental and physical health in the english-speaking $\mathrm{Ca}$ ribbean. Revista Panamericana de Salud Publica, 32(1), 62-69. doi: http://dx.doi.org/10.1590/ S1020-49892012000700010

Pulido-Rull, M. A., Coronel-Villalobos, M., Vera-García, F., \& Barousse-Martínez, T. (2011). Salud física, hábitos alimentarios y ejercicio en estudiantes de licenciatura de la Universidad Intercontinental. Revista Intercontinental de Psicología y Educación, 13, 65-82. Recuperado de http://www.redalyc.org/articulo. oa? id $=80218382005$
Remor, E., \& Pérez-Llantada, M. (2007). La relación entre niveles de la actividad física y la experiencia de estrés y de síntomas de malestar físico. Revista Interamericana de Psicología, 41(3), 313-322. Recuperado de http://www.redalyc. org/articulo.oa? $\mathrm{id}=28441306$

Restrepo, S., Morales, R.; Ramírez, M., López, L., \& Varela, L. (2006). Los hábitos alimentarios en el adulto mayor y su relación con los procesos protectores y deteriorantes de la salud. Revista Chilena de Nutrición, 33(3), 1-18. Recuperado de http://www.redalyc.org/articulo. oa? id=46914636006

Román, C., Ortiz, F., \& Hernández, Y. (2008). El estrés académico en estudiantes latinoamericanos de la carrera de Medicina. Revista Iberoamericana de Educación, 7(46), 1-8. Recuperado de http://rieoei.org/deloslectores/2371Collazo.pdf

Rosario, P., Núñez, J., Salgado, A. González-Pienda, J., Valle, A., Joly, C., et al. (2008). Ansiedad ante los exámenes: Relación con variables personales y familiares. Psicothema, 20(4), 563570. Recuperado de http://www.redalyc.org/ articulo.oa? $\mathrm{id}=72720409$

Rodríguez, N., Ríos, M., Lozano, L., \& Álvarez, M. (2009). Percepción de jóvenes universitarios respecto a su salud: conductas y contexto de riesgo. Enseñanza e Investigación en Psicologia, 14(2), 245-260. Recuperado de http:// www.redalyc.org/articulo.oa?id=29211992003

Ross, C., \& Bird, C. (1994). Sex stratification and health lifestyle: Consequences for men's and women's perceived health. Journal of Health and Social Behavior, 35, 161-178. Recuperado de http://webcache.googleusercontent. com/search?q=cache:EMnqtQDmAuIJ:citeseerx.ist.psu.edu/viewdoc/download\%3Fdoi\%3D10.1.1.475.3462\%26rep\%3Drep1\%26 type $\% 3 \mathrm{Dpdf}+\& \mathrm{~cd}=2 \& \mathrm{hl}=$ es \&ct $=$ clnk $\&$ $\mathrm{gl}=\mathrm{ve}$

Salazar, C., Arrivillaga, M., \& Correa, D. (2003). Creencias sobre la salud y su relación con las prácticas de riesgo o de protección en jóvenes 
universitarios. Colombia Médica, 34, 186-195. Recuperado de http://www.redalyc.org/articulo. oa? id $=28334403$

Salazar-Torres, C., \& Arrivillaga-Quintero, M. (2004). El consumo de alcohol, tabaco y otras drogas, como parte del estilo de vida de jóvenes universitarios. Revista Colombiana de Psicología, 13, 74-89. Recuperado de http://www. redalyc.org/articulo.oa? id $=80401309$

Sanabria, P., González, L., \& Urrego, D. (2007). Estilos de vida saludable en profesionales de salud. Estudio exploratorio. Revista Médica, 15, 207-217. Recuperado de http://www.redalyc. org/articulo.oa? id $=91015208$

Schmidt, M. (2012) Predictors of self-rated health and lifestyle behaviours in Swedish university students. Global journal of health science, 4(4), 1-14. doi: http://dx.doi.org/10.5539/gjhs. $\mathrm{v} 4 \mathrm{n} 4 \mathrm{p} 1$

Sebastiani, R., Pilicioni, M., \& Chiattone, H. (2002). La psicología de la salud latinoamericana: Hacia la promoción de la salud. International Journal of Clinical and Health Psychology, 2(1), 153172. Recuperado de http://www.redalyc.org/ articulo.oa? $\mathrm{id}=33720108$

Seeman, J. (1989). Toward a model of positive health. American Psychologist, 44(8), 1099-1109. doi: http://dx.doi.org/10.1037/0003-066X.44.8.1099

Sierra, J., Jiménez, C., \& Martin, J. (2002). Calidad del sueño en estudiantes universitarios: Importancia de la higiene del sueño. Salud Mental, 25(6), 35-43. Recuperado de http://www.redalyc.org/articulo.oa?id=58262505

Solorzano, M. (2013). Estilos de vida en adolescentes de nivel medio superior de una comunidad semiurbana. Eureka, 10(1), 77-85. Recuperado de http://pepsic.bvsalud.org/pdf/eureka/v10n1/ a09.pdf

Stone, G. (1982). Health psychology: A new journal for a new field. Health Psychology, 1, 1-6. doi: http://dx.doi.org/10.1037/h0090245

Stone, G. (1988). Psicología de la salud: Una definición amplia. Revista Latinoamericana de Psicología, 20, 15-26. Recuperado de http:// www.redalyc.org/pdf/805/80520102.pdf

Taylor, S., Lerner, J., Sage, R., Lehman, B., \& Seeman, T. (2004). Early environment, emotions, responses to stress, and health. Journal of Personality, 72(6), 1365-1393. doi: 10.1111/j.14676494.2004.00300.x

Tibblin, G., Bengtsson, C., Furunes, B., y Lapidus, L. (1990). Symptoms by age and sex: Studies of men and women in gothenburg, sweden. Scandinavian Journal of Primary Health Care, 8, 9-17. doi: 10.3109/02813439008994923

Varela-Mato, V., Cancela, J., Ayan, C. Martín, V., \& Molina, A. (2012). Lifestyle and health among Spanish university students: differences by gender an academic discipline. International Journal of Environmental Research and Public Health, 9, 2728-2741. doi: 10.3390/ijerph9082728

Vargas-Zárate, M., Becerra-Bulla, F., \& Prieto-Suarez, E (2008). Evaluación antropométrica de estudiantes universitarios en Bogotá-Colombia. Revista de Salud Pública, 10(3), 433-442. Recuperado de http://www.scielosp.org/pdf/rsap/ v10n3/v10n3a08.pdf

Zins, M., Bonenfant, S., Carton, M., Coeurei-Pellicer, M., Guéguen, A., Gourmelen, J., et al. (2010). The CONSTANCES cohort: An open epidemiological laboratory. BMC Public Health, 10, 479-487. doi: 10.1186/1471-2458-10-479

\section{Recibido: Enero 15, 2016 Aprobado: Mayo 02, 2016}

